\begin{abstract}
BACKGROUND: Many papers have focused on the importance of granulocytes in the process of reperfusion and ischemia. Most of the clinical studies measured several parameters of this process during and after coronary angiography, without taking into account the effect of the radiographic contrast media (RCM) used during this procedure.

Materials and methods: We performed a randomized patient study $(n=37)$ to evaluate the effect of ionic and non-ionic RCM on granulocyte adhesion during coronary angiography. We also evaluated the influence of the ionicity and osmolarity of the different substances on granulocyte adhesion molecules in in vitro experiments.

Results: The osmolarity of patient serum samples increased from $302 \pm 1$ to $309 \pm 1 \mathrm{mOsm} / \mathrm{kg}(p<0.05)$ after infusion of RCM. The CD11b expression in the samples of the non-ionic RCM treated group increased from $221 \pm 21$ MFI to $377 \pm 30$ MFI $(p<0.05)$ measured as the absolute mean fluorescence intensity (MFI), yet did not alter significantly in the ionic RCM group. In contrast, the in vitro experiments showed a reduction of the CD11b expression from $360 \pm 70$ MFI to $149 \pm 30$ MFI $(p<0.05)$ in the ionic RCM group.

Conclusions: The upregulation of adhesion molecules was significantly reduced in vivo with ionic RCM, while ionic substances caused opposite effects in vitro. This effect should be taken into account when performing leukocyte functional analysis of samples taken during angiography.
\end{abstract}

Key words: Granulocyte; Monocyte, Adhesion molecules, Radiographic contrast media, Angiography

\section{Influence of ionic and non-ionic radiographic contrast media on leukocyte adhesion molecules}

\author{
Guy L. J. Vermeiren ${ }^{1,2}$, Roel Willems ${ }^{3}$, \\ Marc J. Claeys ${ }^{2}$, Chris Vrints ${ }^{2}$, Herman Slegers ${ }^{4}$ and \\ Philippe G. Jorens ${ }^{1, C A}$
}

${ }^{1}$ Department of Intensive Care, ${ }^{2}$ Department of Cardiology ${ }^{3}$ Laboratory of Experimental Hematology, University Hospital of Antwerp, Wilrijkstraat 10, B 2650 Edegem, Belgium, and ${ }^{4}$ Laboratory of Cellular Biochemistry, Department of Biochemistry, University of Antwerp, Belgium

\author{
${ }^{\mathrm{CA}}$ Corresponding author \\ Tel: + 3238213639 ; \\ Fax: + 3238284882 \\ E-mail: Philippe.Jorens@uza.be
}

\section{Introduction}

Leukocyte activation has been shown to play an important role in different inflammatory processes. Moreover, activation of leukocytes has been detected in processes not only characterized or accompanied by inflammation; for example, transplantation medicine, ${ }^{1}$ shock ${ }^{2-4}$ and ischemia/reperfusion processes. $^{5-9}$

One of the most studied mechanisms of leukocyte activation is adhesion. Indeed, in order to migrate into tissue, inflammatory cells need to adhere to the vessel wall. This process is linked to an upregulation of adhesion molecules on both leukocytes and the endothelium. $^{10-12}$

Some studies attempted to analyze whether altered expression of adhesion molecules is detected during ischemia/reperfusion: for instance, in patients submitted to a coronary angiography and percutaneous transluminal coronary angiography. ${ }^{13-15}$ Increased expression of adhesion molecules on leukocytes has been detected in ischemia/reperfusion.
Recently, scientists have become aware of the influence of medication on the activation of leukocytes. Moreover some radiographic contrast media (RCM), which are injected in patients, are known modulators of leukocyte function. ${ }^{16}$ However, the influence on the expression of adhesion molecules has not been previously reported. Therefore, we studied the effects of RCM, both assayed in vitro and in vivo, on the upregulation of granulocyte-adhesion and monocyte-adhesion complexes. We also attempted to discriminate whether these effects were attributable to either RCM ionicity or RCM osmolarity, two characteristics used to classify these agents in four arbitrary groups (Table 1).

\section{Materials and methods}

\section{Patient inclusion}

The inclusion criteria and study protocol were approved by the ethical committee of the University Hospital of Antwerp. Patients with stable angina, 
Table 1. Classification of RCM

\begin{tabular}{lll}
\hline & Low osmolarity & High osmolarity \\
\hline Non-ionic & $\begin{array}{l}\text { loversol } \\
\text { lopromide } \\
\text { lohexol } \\
\text { lomeprol } \\
\text { lonic }\end{array}$ & $\begin{array}{l}\text { loxitalamate } \\
\text { Diatrizoate }\end{array}$ \\
\hline
\end{tabular}

Overview of the classification of RCM. The classification is based on ionic and osmolar properties.

scheduled for a coronary angiography, were considered eligible for inclusion in the study. Patients were only included after informed consent.

Patients were excluded if (i) any sign of inflammation was present (leukocytosis, recent anamnesis of infection, malignancy, etc.), (ii) anti-inflammatory drugs (steroid or non-steroid) were taken on a regular basis, or (iii) the leukocyte count was above $10^{4}$ cells $/ \mathrm{mm}^{3}$ or if a granulocyte percentage above $75 \%$ was found upon analysis.

After inclusion, patients were treated at random with ioxaglate (Hexabrix ${ }^{\circledR}$; Guerbet Codali, Brussels, Belgium) or iopromide (Ultravist ${ }^{\circledR}$; Schering, Diegem, Belgium). In order to avoid interpretation bias, the used RCM was unknown for the cardiologist.

\section{Healthy volunteers}

Several volunteers from the department of biochemistry of the University of Antwerp donated blood for the in vitro experiments. The blood samples were checked for leukocyte number and differentiation. Volunteers were excluded according to the same criteria as the patient population.

\section{Catheterization technique}

Cardiac catheterization was performed using the Seldinger technique. All patients received a 6 Fr arterial sheath in the left femoral artery. All samples were obtained through the arterial sheath. Heparin (5000 IU) was administered at the beginning of the procedure.

\section{Blood sample collection}

Samples were collected using the Vacutainer ${ }^{\circledR}$ technique in $3 \mathrm{ml}$ of ethylenediamine tetraacetic acid (EDTA tubes) (Vacutainer ${ }^{\circledR}$; Beckton Dickinson, Plymouth, UK) and immediately analyzed. Blood samples were collected before angiography (at baseline) and 5 min after the procedure was completed.

Eleven patients agreed to a third sample collection taken $60 \mathrm{~min}$ after completion of the angiography. The latter samples were used to measure the osmolarity and leukocyte activation over a 1-h time course. The samples were analyzed for the mem- brane density of the leukocyte adhesion complex and for serum osmolarity.

\section{In vitro techniques}

Leukocyte adhesion receptor labeling

For a rapid and efficient evaluation of the blood samples, we used the staining procedure described by Macey et al. ${ }^{17}$ Briefly, whole blood $(25 \mu \mathrm{l})$ was incubated in the dark room, with $25 \mu \mathrm{l}$ of $2 \times 10^{-5} \mathrm{M}$ liquid dye solution 751 (LDS 751; Exciton, Dayton, Ohio, USA) at $4^{\circ} \mathrm{C}$ for $15 \mathrm{~min}$. Subsequently the samples were incubated and labeled with $10 \mu \mathrm{l}$ of phyco-erythrine-conjugated anti-CD11b antibody (CD11b-PE) (Beckton and Dickinson, Aalst, Belgium) and fluoresceine-isothiocyanate-conjugated antiCD14 antibody (CD14-FITC) (Beckton and Dickinson). Incubation was performed at room temperature for 10 min. Finally, RPMI-1640 medium (Gibco BRL, Merelbeke, Belgium) buffered with $25 \mathrm{mM}$ of HEPES (Sigma, Bornem, Belgium) was added to a final volume of $500 \mu \mathrm{l}$ and analyzed on a flow cytometer (FACScan; Beckton Dickinson). CD11b expression, quantified as the mean fluorescence intensity, was measured on granulocytes and monocytes, discriminated by scatter profile and CD14 expression.

\section{Serum osmolarity measurement}

Osmolarities were measured using an automated osmometer (Cryomatic ${ }^{\circledR}$, Model 3C2; Advanced Instruments Inc., Needham Heights, Massachusetts, USA).

\section{In vitro activation assay}

Blood samples were collected from healthy volunteers $(n=5)$. The samples were incubated with the two types of RCM used in the catheterization unit of the University Hospital: ioxaglate (HEXABRIX ${ }^{\circledR}$; Guerbet Codali) and iopromide (ULTRAVIST ${ }^{\circledR}$; Schering).

After injection in patients, RCM dilutions were estimated during the patient trial, and physiologically acceptable dilutions (290, 310, 330 and 350 mOsm/ $\mathrm{kg}$ ) were tested.

As RCM may have both ionic and osmotic properties, we supplemented phosphate-buffered saline (PBS) solutions (Dulbecco's Phosphate Buffered Saline; Gibco BRL) with either saline or sucrose. Saline solutions have osmotic and highly ionic properties, whereas sucrose is a non-ionic compound. We used both solutions in order to prepare solvents with the same range of osmolarities as RCM. Glucose (G), saline (S), ioxaglate (IX) and iopromide (IP) were tested in the following osmolarities: 290, 310, 330 and $350 \mathrm{mOsm} / \mathrm{kg}$. The Blank PBS was tested in $280 \mathrm{mOsm} / \mathrm{kg}$.

Blood samples of healthy volunteers were collected in $4 \mathrm{ml}$ EDTA tubes (Vacutainer ${ }^{\circledR}$; Beckton 
Dickinson) and were divided in $200 \mu$ l aliquots. Each aliquot was incubated with $4 \mathrm{ml}$ of lysis buffer (155 $\mathrm{mM} \mathrm{NH} 4 \mathrm{Cl}, 10 \mathrm{mM} \mathrm{KCO}_{3}, 0.1 \mathrm{mM} \mathrm{Na} 2$ EDTA) for 5 min at room temperature.

Subsequently the samples were centrifuged for 15 min at $400 \mathrm{~g}$. The supernatant was discarded and the cell pellet was resuspended in either PBS or one of the osmotic solutions. The incubation with the osmotic solutions was maintained at $37^{\circ} \mathrm{C}$ during 20 min, an incubation time that induced maximal granulocyte activation as shown in preliminary experiments (data not shown).

The cell suspension was incubated with $10 \mu \mathrm{l}$ of CD11b-PE and CD14-FITC during $15 \mathrm{~min}$, after which the excess of non-ligated antibody was removed by centrifugation. After resuspension in $500 \mu \mathrm{lBS}$, flow cytometric analysis for adhesion molecules was performed.

\section{Statistical analysis}

Statview $^{\circledR}$ software (version 5.0; SAS, Cary, North Carolina, USA) was used for all statistical calculations. Corrections for non-parametric analysis were performed (i.e. Mann-Whitney, Kruskal-Wallis and Spearman rank correlation analysis).

Whenever mean values and their range are mentioned, we used the standard error of the mean.

\section{Results}

\section{Patient study}

A total of 37 patients was included in the study. The population consisted of 19 male subjects (age 61.1 \pm 8.5 years) and 18 female subjects (age 66.8 \pm 11.5 years). Mean fluorescence signals at baseline showed no difference between the ioxaglate-treated and the iopromide-treated patients (Mann-Whitney $p=0.72$ ) (Fig. 1).

Analysis of the samples revealed a significant increase in CD11b intensity as well as osmolarity after $5 \mathrm{~min}$. A subpopulation of 21 patients agreed to donate a third sample collected $60 \mathrm{~min}$ after injection of RCM. The results are presented in Fig. 1. Iopromide significantly elevated the CD11b-receptor density in the granulocyte population, $5 \mathrm{~min}$ after administration $(p<0.001)$. This effect disappeared after $60 \mathrm{~min}$, and the fluorescence values returned to baseline $(p=0.81)$. The monocyte population showed an analogous trend to elevate the CD11breceptor density $(p=0.06)$. Monocyte activation also seemed to persist for more than $60 \mathrm{~min}$. Patients treated with ioxaglate showed no significant difference in CD11b-receptor density over time.

The osmolarity of the samples was determined. The measured baseline osmolarity was measured at
$302 \pm 1 \mathrm{mOsm} / \mathrm{kg}$. Samples taken $5 \mathrm{~min}$ after RCM administration showed a significant $(p=0.003)$ elevation of the serum osmolarity $(309 \pm 1 \mathrm{mOsm} / \mathrm{kg})$. The osmolarity of the samples at baseline and the samples taken $60 \mathrm{~min}$ after RCM administration $(304 \pm 2 \mathrm{mOsm} / \mathrm{kg})$ showed no statistical significant difference. The results are presented in Fig. 2.

\section{In vitro study}

To validate the results of the clinical study, we studied the effects of RCM on granulocytes and monocytes in vitro. For convenience, the in vitro experiments were limited to the two RCMs used in our hospital. Blood samples were collected from healthy volunteers $(n=5)$.

As the maximal osmolarity in patients was estimated to be $309 \pm 1 \mathrm{mOsm} / \mathrm{kg}$, we used solutions with an osmolarity varying between 290 and 350 $\mathrm{mOsm} / \mathrm{kg}$ to study the activation of granulocytes in osmotic media.

To properly evaluate the effect of the solutions we normalized the mean fluorescence values. Granulocytes can exhibit an elevated level of the initial CD11b-receptor density. This form of elevated activity is denoted as 'granulocyte priming. ${ }^{18}$ As the maximal activity (degranulation or upregulation of CD11b) of the granulocyte will not change, a primed granulocyte will have an apparently decreased activity. We normalized the values in such a way that the blank value (PBS without any additives) was correlated with $0 \%$ activation and the maximal measured value was expressed as 100\% activation.

The results clearly showed a difference between granulocyte and macrophage responses, as observed in the clinical study (Fig. 3). CD11b-receptor density on monocytes is relatively stable, regardless of the degree of ionicity or osmolarity of the RCM. Granulocytes, on the contrary, seem to be more susceptible to these factors.

To evaluate the effect of osmolarity on the activation of granulocytes and monocytes, we prepared a concentration gradient of sucrose-enriched PBS solutions. In order to rule out possible additive effects of ionicity, a comparison was also made with analogous solutions of PBS. Using a saline or sucrose gradient, we could not establish any influence of either the ionicity or osmolarity (Fig. 3), even at $350 \mathrm{mOsm} / \mathrm{kg}$, which is well above the measured osmolarity in vivo.

Next to the influence of ionicity and osmolarity we also tested the RCMs. In this way we could evaluate the substance-related effects, regardless of their ionic and osmolar properties. Ioxaglate seems to exert a significant downregulating effect on CD11b-receptors at higher osmolarities; for example, at 330-350 $\mathrm{mOsm} / \mathrm{kg}$ (hypothesis of no difference).

The effect is most prominent on the activity of granulocytes. Using a Kruskal-Wallis analysis, we 


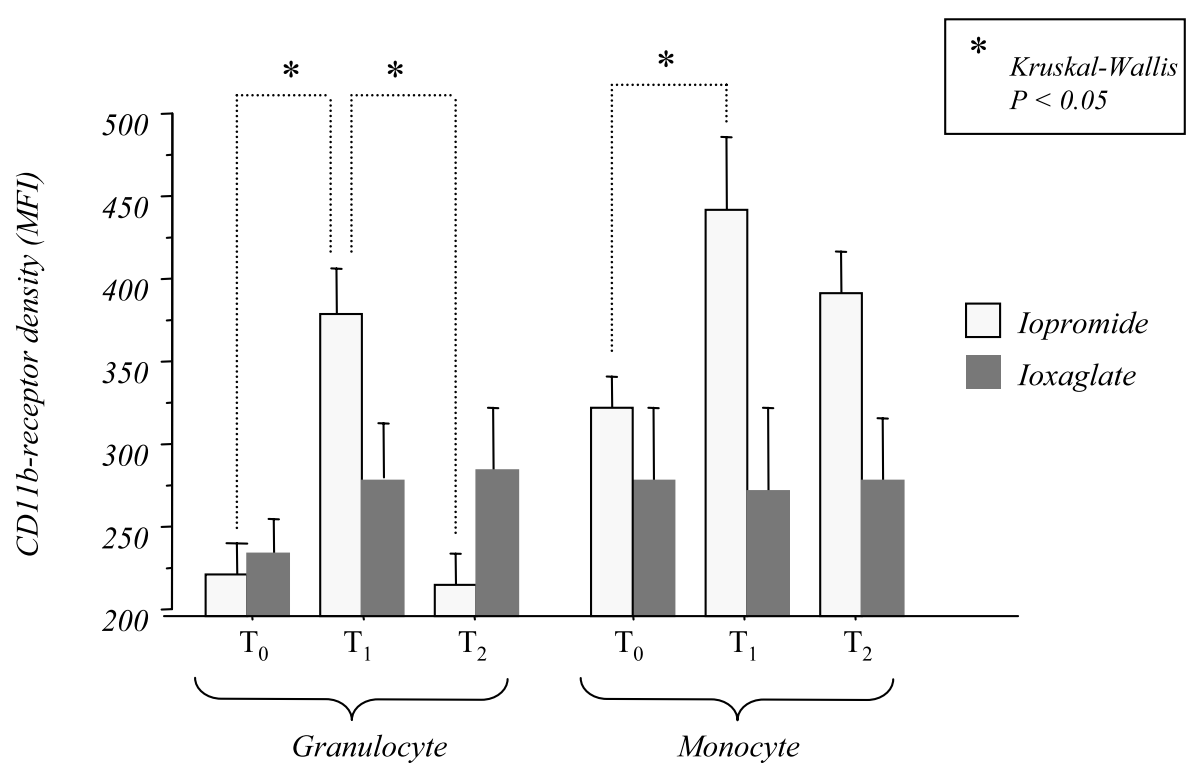

FIG. 1. Changes in CD11b levels on leukocytes after RCM infusion. The mean fluorescence intensity (MFI) of anti-CD11b antibody on granulocytes and monocytes during infusion of iopromide and ioxaglate. $T_{0}$, sample taken before the initiation of the procedure; $T_{1}$, sample taken 5 min after infusion of $R C M ; T_{2}$, sample taken 60 min after the infusion of RCM. * Statistically significant differences $(p<0.05)$.

observe a significant difference $(p<0.05)$ between ioxaglate and iopromide (Fig. 3). The effect can be described as an inhibition of the upregulation of CD11b-receptors at the cell membrane. Iopromide does not show such an effect.

To correlate the effect of the ioxaglate concentration (in serum) to its inhibitory potential, a regression analysis with Spearman rank correction was performed (Fig. 4). This analysis produces a spearman rho of 0.7 with an estimated $r^{2}$ value of 0.48 . No other significant correlations could be found.

\section{Discussion}

RCM are frequently used for both diagnostic procedures and clinical research. Altering the X-ray absorption of tissues, vessels and cavities enables the clinician to visualize anatomical structures in the organism, which provides information about organ mass and function, such as revascularization of the coronary arteries.

However, many unwanted pharmacological effects are observed after the use of RCM, ranging from

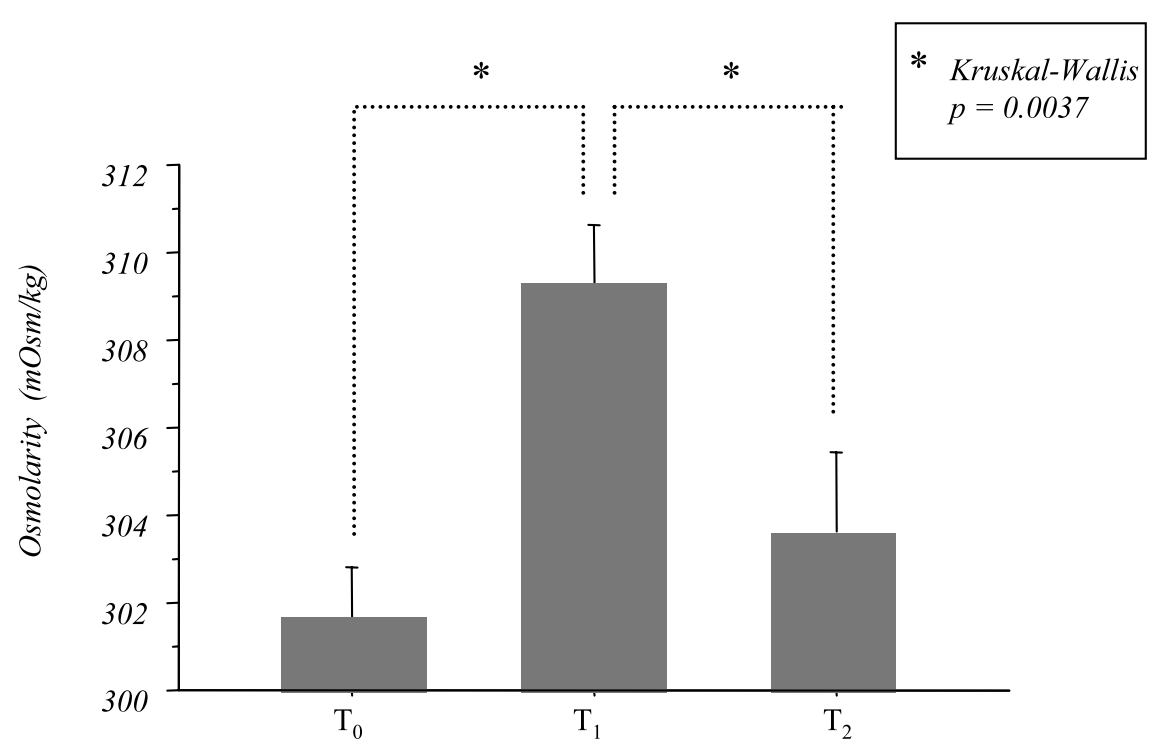

FIG. 2. Transient increase in serum osmolarity after iopromide infusion. $T_{0}$, sample taken before the initiation of the procedure; $T_{1}$, sample taken 5 min after infusion of RCM; $T_{2}$, sample taken 60 min after the infusion of RCM. * Statistically significant differences $(p<0.05)$. 


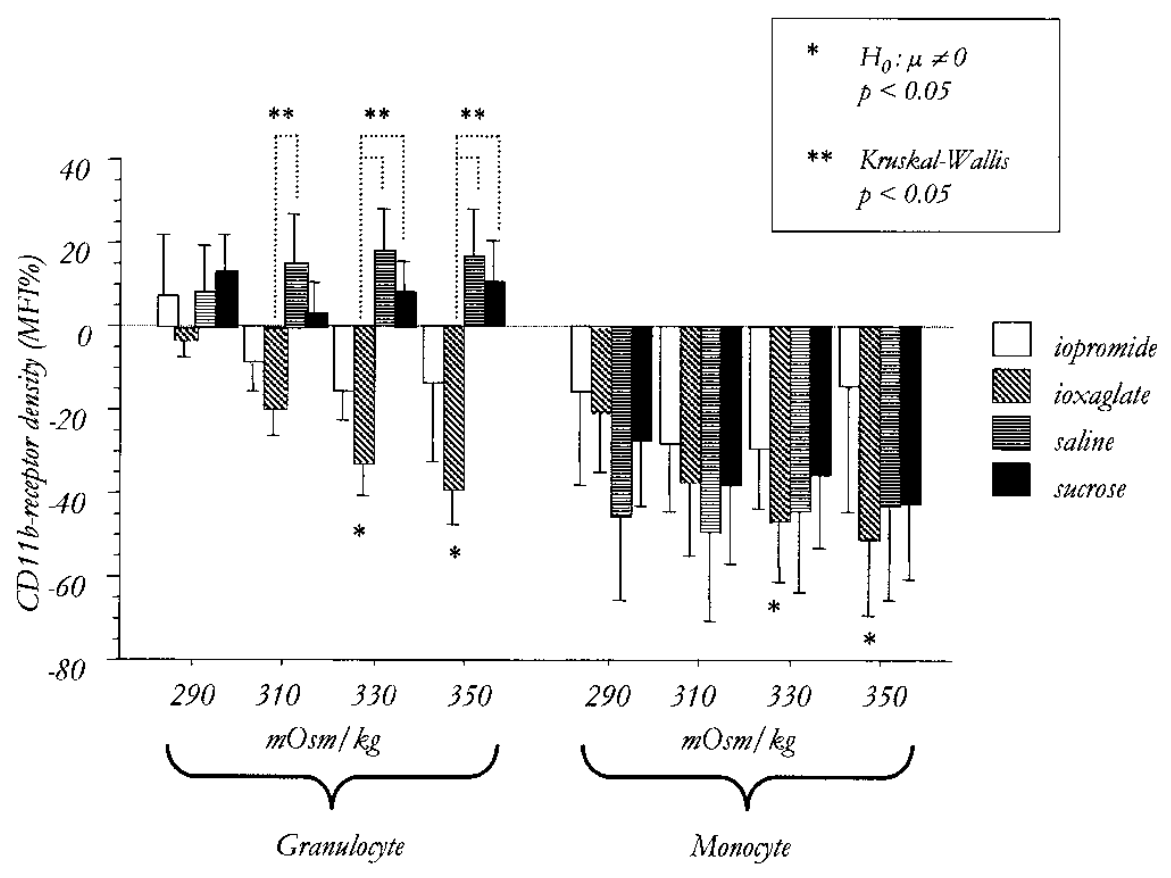

FIG. 3. 'In vitro' effect of osmolarity on CD11b expression. The effect of different solutions of iopromide, ioxaglate, sucrose and saline, with increasing osmolarity, on granulocytes and monocytes. ${ }^{*}$ Statistical significant difference from zero $(p<$ 0.05). ** Significant statistical $(p<0.05)$ 'between group' difference. CD11b-receptor density is expressed in\% MFI, a value normalized for blank and maximal stimulation.

disruption of the blood-brain barrier ${ }^{19-21}$ to severe anaphylactic reactions. ${ }^{22,23}$ The latter is well studied and many precautions can be taken to prevent ${ }^{24}$ or treat $^{25}$ its aftermath. RCMs affect different physiological systems such as the coagulation and arachidonic acid system and may interact with many human cells.

Several publications have covered the problem of an elevated risk for thrombosis with non-ionic substances. ${ }^{26-28}$ In vitro experiments have shown that platelets more readily degranulate in the presence of non-ionic substances. ${ }^{29}$
In our clinical study we observed an elevated CD11b expression after iopromide injection. This 'activating' effect of iopromide, a member of the non-ionic RCM group, has not yet been described in the literature. An increased adhesion to nylon fibers was described by Lang and Lang. ${ }^{30}$

Yet ioxaglate does not affect the CD11b expression in vivo, in contrast to the findings of Feldman et al ${ }^{31}$ The question remains of whether the ionicity or the osmolarity of the substance is actually responsible for this effect.

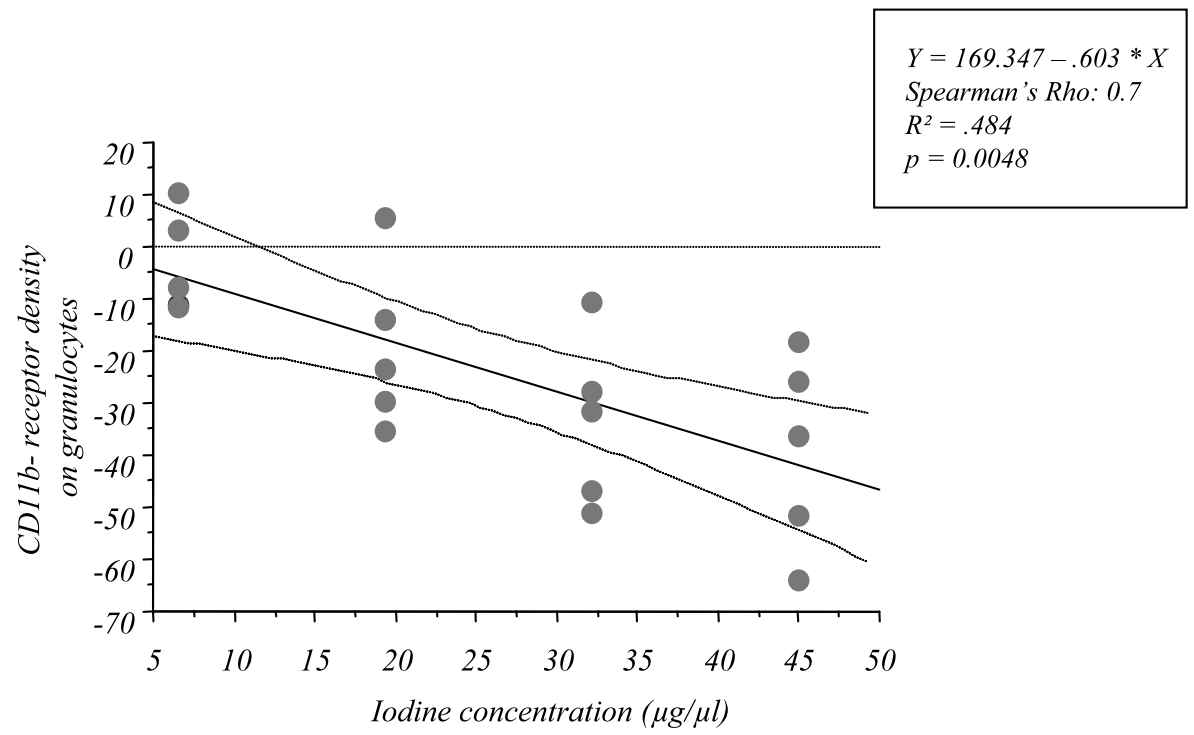

FIG. 4. 'In vitro' effect of ioxaglate concentration on CD11b expression. Linear regression analysis for CD11b expression in\% MFI (normalized for blank and maximal value) and iodine concentration of ioxaglate $(\mu \mathrm{g} / \mu \mathrm{l})$. Linear regression was corrected by Spearman rank calculation. 
The in vitro experiment clearly showed that neither osmolarity nor ionicity affected the activity of the leukocytes in our experiment. Neither could we observe a response to an elevation of the ionicity. Sodium chloride could not evoke any response on the leukocytes. Thus, ionicity alone cannot account for the protective effects as described previously in the literature. ${ }^{32}$

From the results of the RCM in vitro experiments we may conclude that ioxaglate exerts a downregulating effect on the adhesion molecules at higher osmolarities. As neither osmolarity, nor ionicity is responsible for this effect, the in vitro effect is probably due to the ioxaglate concentration. This in vitro effect is more evident on granulocytes than on monocytes and has not yet been previously described. Iopromide showed no significant effect on the leukocytes whatsoever.

At first glance this seems rather contradictory with regard to the in vivo experiment. This might well mean that ioxaglate neutralizes a 'catheterizationassociated' activation, whereas the protective effect of iopromide is too weak to exert any effect at all.

The results of this study clearly indicate that ionic RCM may well inhibit leukocyte activation. The question remains of whether the choice between ionic and non-ionic RCM is indeed crucial. Several authors implied that the choice between different RCMs is not so important. Regardless of their ionic and/or osmotic properties the overall clinical outcome (in terms of major adverse cardiac events) remains unchanged. ${ }^{33,34}$ Other trials support the use of ionic RCM as being more favorable. ${ }^{35}$

Several other granulocyte functions are also influenced after contact with RCM, but conflicting data have been published. Following incubation of granulocyte with RCM, both a potentiation and an inhibition of exocytosis (i.e. the release of proteases by degranulation) has been reported. ${ }^{36}$ Both inhibition $^{37}$ and increased chemotaxis ${ }^{38}$ have also been observed. Most papers indicated an inhibition of phagocytosis. ${ }^{39}$ In general, ionic media are more inhibitory than non-ionic.

Many publications measured the membrane-associated CD11b immediately after angiography or percutaneous transluminal coronary angiography, yet they do not specify the RCM used in their protocol. ${ }^{40-42}$ The observed in vivo effects on granulocyte functions have been described insignificant and transient. However, they should be taken into account when evaluating the early activation in vivo during procedures of ischemia/reperfusion. Caution is warranted for the interpretation of experimental results measured within $30 \mathrm{~min}$ of $\mathrm{RCM}$ administration.

ACKNOWLEDGEMENTS. The authors would like to acknowledge all nursing and technical personnel of the catheterization unit for their logistic support. Furthermore, they would like to express their gratitude for the volunteers of the laboratory for Cellular Biochemistry for their continuous donation of blood samples during the preliminary phase of the project. In particular, the authors wish to thank Miss M. Dendooven, M.Sc. for her kind assistance. Finally the authors wish to acknowledge F. De Roeck, M.D. for providing us with sufficient amounts of RCM. G.V. was supported by a grant from Pfizer.

\section{References}

1. Akyurek ML, Funa K, Wanders A, Larsson E, Fellstrom BC. Expression of CD11b and ICAM-1 in an in vivo model of transplant arteriosclerosis. Transpl Immunol 1995; 3: 107-113.

2. Childs EW, Wood JG, Smalley DM, Hunter FA, Cheung LY. Leukocyte adherence and sequestration following hemorrhagic shock and total ischemia in rats. Shock 1999; 11: 248-252.

3. Mayumi T, Chan CK, Clemens MG, Bulkley GB. Zonal heterogeneity of hepatic injury following shock/resuscitation: relationship of xanthine oxidase activity to localization of neutrophil accumulation and central lobular necrosis. Shock 1996; 5: 324-332

4. Rhee P, Burris D, Kaufmann C, et al. Lactated Ringer's solution resuscitation causes neutrophil activation after hemorrhagic shock. $J$ Trauma 1998; 44: 313-319.

5. Go LO, Murry CE, Richard VJ, Weischedel GR, Jennings RB, Reimer KA. Myocardial neutrophil accumulation during reperfusion after reversible or irreversible ischemic injury. Am J Physiol 1988; 255: H1188-H1198.

6. Ernst E, Hammerschmidt DE, Bagge U, Matrai A, Dormandy JA. Leukocytes and the risk of ischemic diseases. JAMA 1987; 257: $2318-$ 2324

7. de-Lorgeril M, Rousseau G, Basmadjian A, St-Jean G, Tran DC, Latour JG. Spacial and temporal profiles of neutrophil accumulation in the reperfused ischemic myocardium. Am J Cardiovasc Pathol 1990; 3: $143-154$.

8. Komatsu H, Koo A, Ghadishah E, et al. Neutrophil accumulation in ischemic reperfused rat liver: evidence for a role for superoxide free radicals. Am J Physiol 1992; 262: G669-G676.

9. Vermeiren GL, Claeys MJ, Van Bockstaele D, Grobben B, Slegers H, Bossaert L, Jorens PG. Reperfusion injury after focal myocardial ischaemia: polymorphonuclear leukocyte activation and its clinical implications. Resuscitation 2000; 45: 35-61.

10. Lefer AM. Role of selectins in myocardial ischemia-reperfusion injury. Ann Thorac Surg 1995; 60: 773-777.

11. Zhao ZQ, Lefer DJ, Sato H, Hart KK, Jefforda PR, Vinten-Johansen J. Monoclonal antibody to ICAM-1 preserves postischemic blood flow and reduces infarct size after ischemia-reperfusion in rabbit. J Leukoc Biol 1997; 62: 292-300

12. Albertine KH, Weyrich AS, Ma XL, Lefer DJ, Becker LC, Lefer AM. Quantification of neutrophil migration following myocardial ischemia and reperfusion in cats and dogs. J Leukoc Biol 1994; 55: 557-566.

13. de-Servi S, Mazzone A, Ricevuti G, et al. Expression of neutrophil and monocyte CD11B/CD18 adhesion molecules at different sites of the coronary tree in unstable angina pectoris. Am J Cardiol 1996; 78: $564-$ 568.

14. Inoue T, Sakai Y, Hoshi K, Yaguchi I, Fujito T, Morooka S. Lower expression of neutrophil adhesion molecule indicates less vessel wall injury and might explain lower restenosis rate after cutting balloon angioplasty. Circulation 1998; 97: 2511-2518.

15. Kowalski J, Kosmider M, Pawlicki L, et al. Complement activates neutrophils during PTCA procedure in patients with unstable angina pectoris. Int J Cardiol 1997; 58: 229-240.

16. Rasmussen $\mathrm{F}$. The influence of radiographic contrast media on some granulocyte functions. Acta Radiol 1998; 419S: 7-35.

17. Macey MG, McCarthy DA, Newland AC. The ex vivo function and expression of function-associated antigens of peripheral blood neutrophils and monocytes. Exp Hematol 1994; 22: 967-972.

18. Bainton DF, Miller LJ, Kishimoto TK, Springer TA. Leukocyte adhesion receptors are stored in peroxidase-negative granules of human neutrophils. J Exp Med 1987; 166: 1641-1653.

19. Casady RL, Kitten GT, Bradley IM, Sterrett PR. Sites of cerebrovascular injury induced by radiographic contrast media. Am J Anat 1978; 153: $477-482$.

20. Hayakawa K, Yamashita K, Mitsumori M, Nakano Y. Blood-brain barrier injury following intracarotid injection of radiographic contrast media. In vivo quantification using magnetic resonance imaging and Gd-DTPA. Acta Radiol 1990; 31: 203-208.

21. Wilson AJ, Evill CA, Pope LM, Sage MR. Chemotoxicity testing of nonionic radiographic contrast media against the blood-brain barrier. Acad Radiol 1996; 3(Suppl 2): S331-S332.

22. Langton JA, Magee P, Sedgwick J, Sutton R. Anaphylaxis following 'Hexabrix' during routine coronary angiography. Eur J Radiol 1988; 8: $60-61$.

23. Hoem NO, Briseid G, Ekelund S, Salvesen S. Effects of intravenous radiographic contrast media on the blood pressure and on factors of the contact activation system in the rat. Acta Pharmacol Toxicol (Copenh) 1986; 59: 189-194 
24. Ring J, Rothenberger KH, Clauss W. Prevention of anaphylactoid reactions after radiographic contrast media infusion by combined histamine $\mathrm{H} 1$ - and H2-receptor antagonists: results of a prospective controlled trial. Int Arch Allergy Appl Immunol 1985; 78: 9-14.

25. Mueller PG. Emergency treatment of anaphylactoid reactions to radiographic contrast materials. Md Med J 1985; 34: 277-279.

26. Fay WP, Parker AC. Effects of radiographic contrast agents on thrombin formation and activity. Thromb Haemost 1998; 80: 266-272.

27. Melton LG, Dehmer GJ, Gabriel DA. Alterations in fibrin detected in coronary sinus blood after heparin and coronary angiography with a nonionic contrast agent (iohexol). Am J Cardiol 1998; 82: 295-298.

28. Hill JA, Grabowski EF. Relationship of anticoagulation and radiographic contrast agents to thrombosis during coronary angiography and angioplasty: are there real concerns? Cathet Cardiovasc Diagn 1992; 25: 200-208.

29. Chronos NA, Goodall AH, Wilson DJ, Sigwart U, Buller NP. Profound platelet degranulation is an important side effect of some types of contrast media used in interventional cardiology. Circulation 1993; 88 $2035-2044$.

30. Lang EV, Lang JH. Effect of contrast agents on adherence characteristics of granulocytes. Invest Radiol 1991; 26(Suppl 1): S29-S32.

31. Feldman LJ, Chollet-Martin S, Himbert D, Juliard J, Pasquier C, Elbim C, Steg PG. Modulation of the expression of the granulocyte adhesion molecule, CR3, by percutaneous transluminal coronary angioplasty and contrast media. Invest Radiol 1994; 29: 313-318.

32. Georgsen J, Rasmussen F, Pedersen JO. Granulocyte chemotaxis. Influence of radiographic contrast media on the chemoattractive properties of serum. Acta Radiol 1990; 31: 531-536.

33. Bertrand ME, Esplugas E, Piessens J, Rasch W. Influence of a nonionic, iso-osmolar contrast medium (iodixanol) versus an ionic, low-osmolar contrast medium (ioxaglate) on major adverse cardiac events in patients undergoing percutaneous transluminal coronary angioplasty a multicenter, randomized, double-blind study. Circulation 2000; 101: 131 136.
34. Schrader R, Esch I, Ensslen R, et al. A randomized trial comparing the impact of a nonionic (Iomeprol) versus an ionic (Ioxaglate) low osmolar contrast medium on abrupt vessel closure and ischemic complications after coronary angioplasty. J Am Coll Cardiol 1999; 33: 395-402.

35. Grines CL, Schreiber TL, Savas V, et al. A randomized trial of low osmolar ionic versus nonionic contrast media in patients with myocardial infarction or unstable angina undergoing percutaneous transluminal coronary angioplasty. J Am Coll Cardiol 1996; 27: 1381-1386.

36. Georgsen J, Rasmussen F, Antonsen S, Larsen ML. Influence of radiographic contrast media on granulocyte enzymes and complement during uncomplicated urographies. Eur I Radiol 1991; 12: 63-66.

37. Carr DH, Walker AC, White RG. Effects of radiographic contrast media on leukocyte locomotion. Invest Radiol 1981; 16: 133-140.

38. Till G, Rother U, Gemsa D. Activation of complement by radiographic contrast media: generation of chemotactic and anaphylatoxin activities. Int Arch Allergy Appl Immunol 1978; 56: 543-550.

39. Farrow R, Roobottom CA, Wells IP, Hurlock N. Effects of radiographic contrast media on leukocyte phagocytosis. Acad Radiol 1994; 1: $249-$ 252 .

40. Ikeda $\mathrm{H}$, Nakayama $\mathrm{H}$, Oda $\mathrm{T}$, et al. Neutrophil activation after percutaneous transluminal coronary angioplasty. Am Heart J 1994; 128: $1091-1098$

41. Serrano Jr CV, Ramires JA, Venturinelli M, et al. Coronary angioplasty results in leukocyte and platelet activation with adhesion molecule expression. Evidence of inflammatory responses in coronary angioplasty. J Am Coll Cardiol 1997; 29: 1276-1283

42. Baj Z, Kowalski J, Kantorski J, Pokoca L, Kosmider M, Pawlicki L, Tchorzewski $H$. The effect of short-term myocardial ischemia on the expression of adhesion molecules and the oxidative burst of coronary sinus blood neutrophils. Atherosclerosis 1994; 106: 159-168.

\section{Received 3 June 2003}

Revised 1 July 2003 


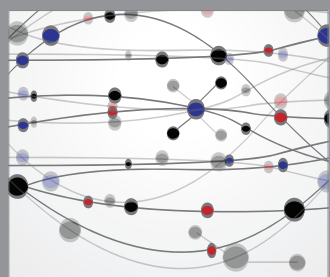

The Scientific World Journal
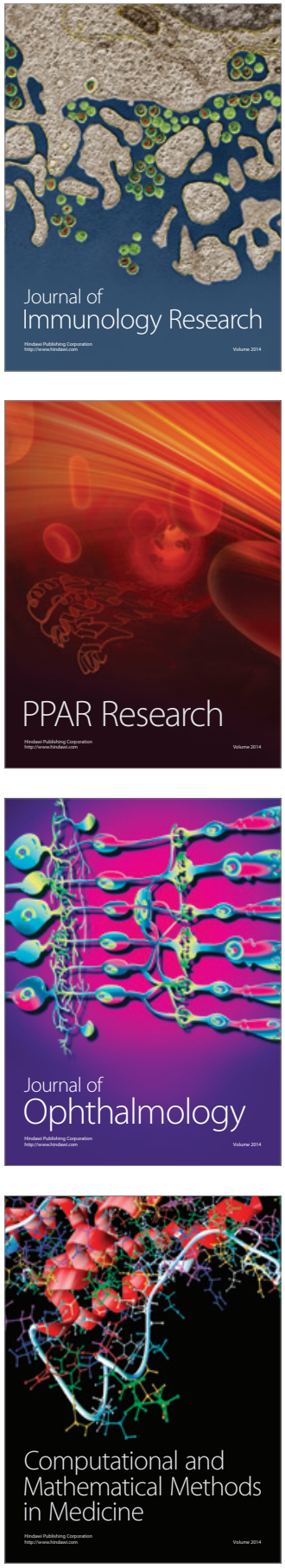

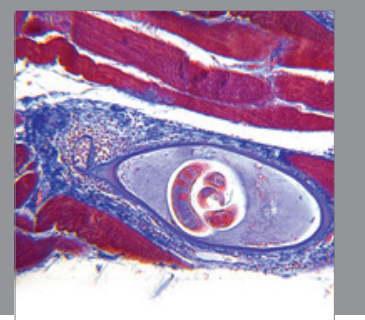

Gastroenterology

Research and Practice
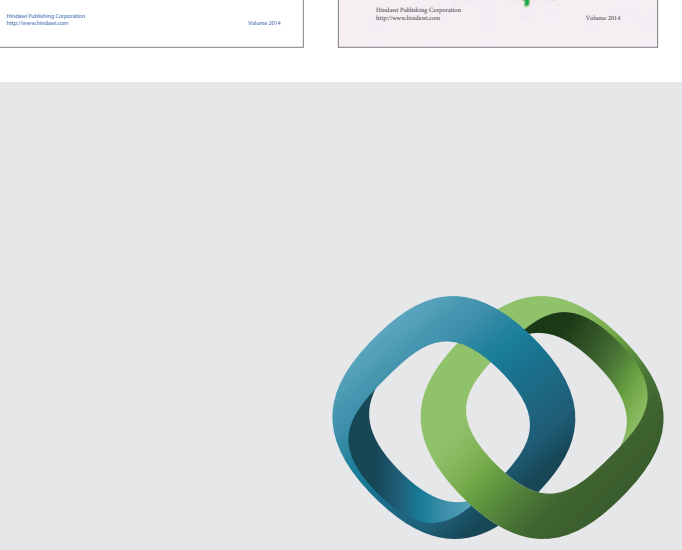

\section{Hindawi}

Submit your manuscripts at

http://www.hindawi.com
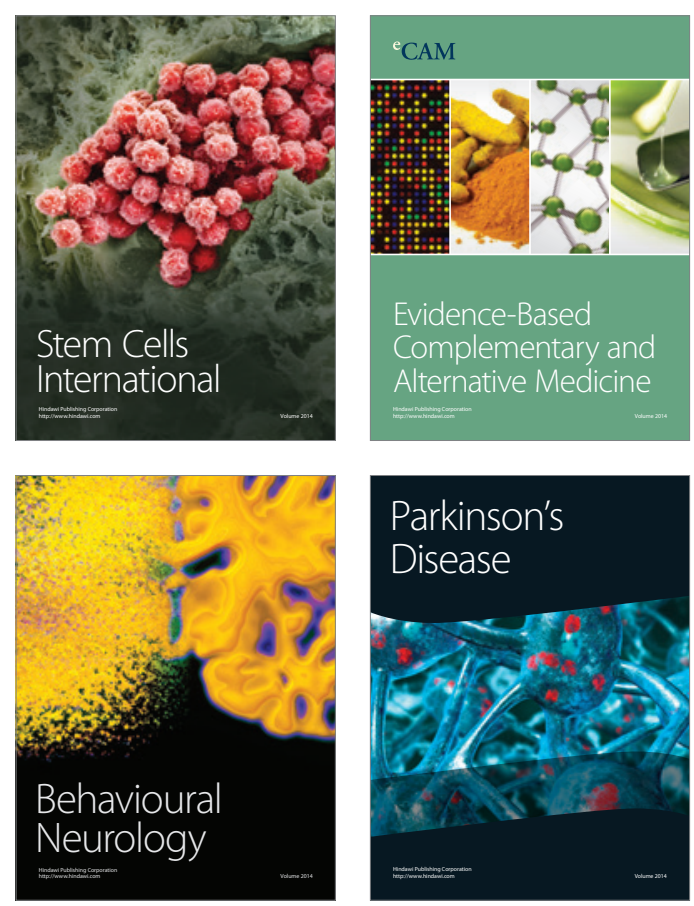

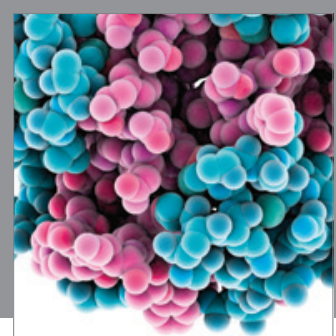

Journal of
Diabetes Research

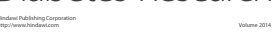

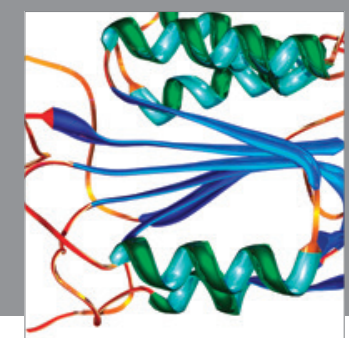

Disease Markers
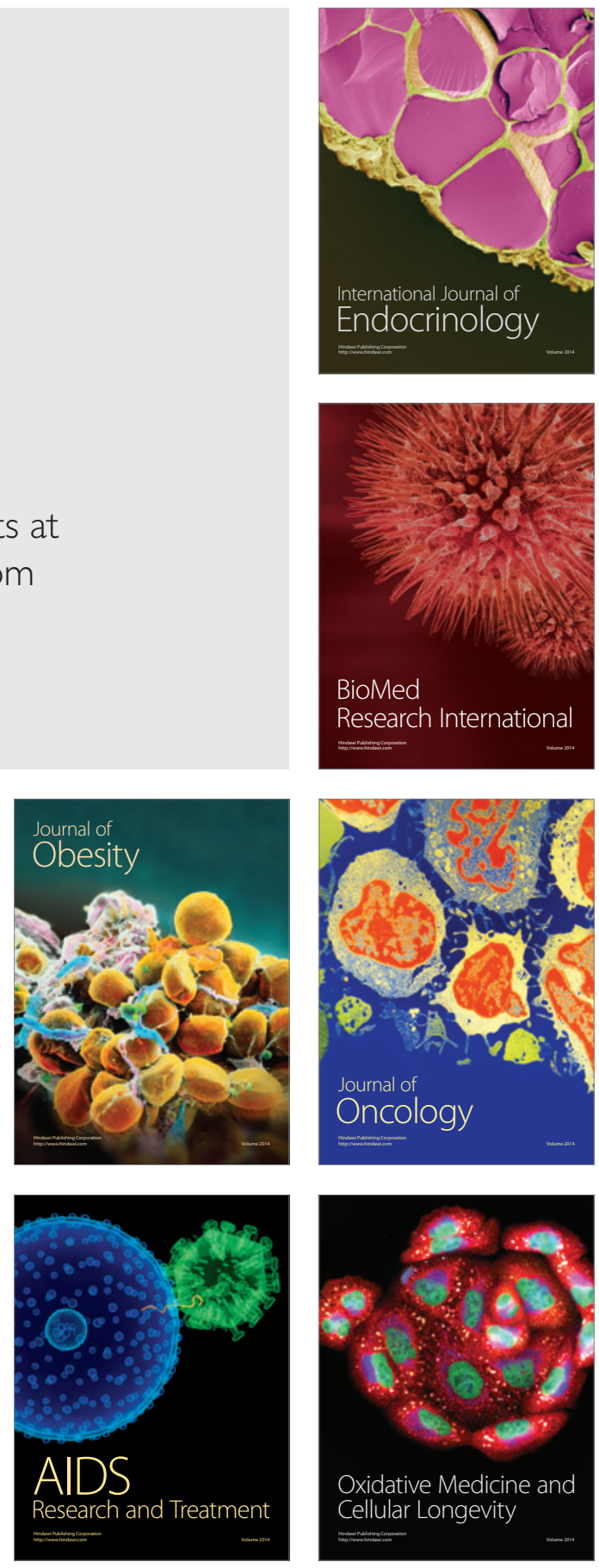